\title{
THERMODYNAMICS OF A CLOSED-CYCLE GAS FLOW SYSTEM FOR COOLING A HTc DC-SQUID MAGNETOMETER
}

P.J. van den Bosch, H.J.M. ter Brake, G.C. van den Eijkel, J.P. Boelens, H.J. Holland, J.F.C. Verberne, and H. Rogalla

University of Twente, Department of Applied Physics, P.O. Box 217, 7500 AE Enschede, The Netherlands

\begin{abstract}
A multichannel high- $T_{c}$ dc-SQUID based heart-magnetometer is currently under development in our laboratory. The system is cooled by a cooler that, due to its magnetic interference, has to be separated from the SQUID unit. In the present prototype system a closed-cycle gas flow was chosen as the interface between the SQUID unit and the cooler (a Leybold Heraeus RG 210). In this paper the prototype system is shortly described, its thermodynamic behaviour is considered and simulations are compared with experimental results.
\end{abstract}

\section{INTRODUCTION}

Nowadays high- $T_{c}$ dc-SQUIDs are available that operate at relatively high temperatures. Therefore, smallscale cryocoolers can be applied for cooling a simple-to-use SQUID-based magnetometer. We constructed a closed-cycle gas flow system for cooling a SQUID-based magnetometer for heart measurements incorporating a Leybold Heraeus RG 210 cooler [1]. We plan to use the experience obtained with this configuration in the design of smaller future systems, based on one or more miniature Stirling cryocoolers [2]. For that purpose a thermodynamic model of the system is under development and in this paper we present the first results. The cold head unit and the rest of the system are modelled separately. Simulations are compared with experimental results.

\section{SYSTEM DESCRIPTION}

The closed-cycle gas flow system incorporates a LH RG 210 cooler that, due to its magnetic interference, has to be separated from the SQUID unit. Helium gas is cooled by the cooler, transported through a $2.5 \mathrm{~m}$ long coaxial gas transfer line, and after that through a heat exchanger on which SQUIDs can be installed (see Fig. 1). Because the gas flow pump has to operate at room temperature, a counterflow heat exchanger was incorporated. Five separate parts can thus be distinguished: the cryocooler unit (consisting of the cooler with heat exchangers mounted on the two cold heads), the gas line unit (four coaxial tubes for the supply and return gas and for thermal insulation), the SQUID unit (a SQUID-plate heat exchanger and a radiation-shield heat exchanger), the counterflow heat exchanger (two $1 \mathrm{~m}$ long coaxial tubes), and the gas flow controller (a pump, mass flow controllers and buffers). The length of the gas circuit of the system without the gas flow controller is about $11 \mathrm{~m}$. This system has been constructed and tested, and extensively described elsewhere [1]. The temperatures are measured at ten positions for different mass flows (between $2 \cdot 10^{-6}$ and $3.2 \cdot 10^{-5} \mathrm{kgs}^{-1}$ ). The lowest obtainable SQUID plate temperature is $31 \pm 2 \mathrm{~K}$ that can be reached in roughly $2-3$ hours with an optimal mass flow of $6 \cdot 10^{-6} \mathrm{~kg} / \mathrm{s}$. This lowest temperature is for a large part determined by the counterflow heat exchanger.

\section{THERMODYNAMICS AND SIMULATIONS}

In all system elements heat is transferred between the gas and the surrounding material and between the latter material and its environment. The energy balances (in $\mathrm{Wm}^{-1}$ ) for the elements can be written as a 
pair of coupled differential equations: eq. (1) for the gas and eq. (2) for the material:

$$
\begin{aligned}
& M C_{V} \frac{\partial T}{\partial t}+\dot{m} C_{p} \frac{\partial T}{\partial x}=h P\left(T_{m}-T\right) \\
& M_{m} C \frac{\partial T_{m}}{\partial t}=-h P\left(T_{m}-T\right)+Q_{e}\left(T_{e}, T_{m}\right) .
\end{aligned}
$$

Here $\mathrm{M}=$ mass of the gas per unit length $\left(\mathrm{kgm}^{-1}\right), \dot{\mathrm{m}}=$ mass flow of the gas $\left(\mathrm{kgs}^{-1}\right), \mathrm{T}=$ temperature of the gas $(\mathrm{K}), \mathrm{T}_{\mathrm{m}}=$ temperature of the material $(\mathrm{K}), \mathrm{c}=$ heat capacity $\left(\mathrm{Jkg}^{-1} \mathrm{~K}^{-1}\right), \mathrm{h}=$ heat transfer coefficient $\left(\mathrm{Wm}^{-2} \mathrm{~K}^{-1}\right)$ in general depending on temperatures, $\mathrm{P}=$ perimeter of the gas channel $(\mathrm{m}), \mathrm{M}_{\mathrm{m}}=$ mass of the channel material per unit length $\left(\mathrm{kgm}^{-1}\right), \mathrm{Q}_{\mathrm{e}}=$ external heat load per unit length $\left(\mathrm{Wm}^{-1}\right)$ depending on the environmental temperature $T_{e}(K)$ and on $T_{n}$. The heat capacity $c_{v}$ originates from the relation between internal energy and temperature for ideal gases.

Simplifications:

- Diffusion of heat is only taken into account in the cold-head unit. In the other elements the heat conductivity of the material is sufficiently low. Diffusion in the gas is neglected compared to the convection term (large Péclet number);

- Radial heat conduction in the gas channel material is ideal (Biot number «1);

- The gas is considered as an incompressible fluid (low Mach number).

As a first step in the development of a thermodynamic model of the complete system, we separated the cold-head unit from the rest. First, the cold-head unit is considered and after that a first model of the rest of the system is presented.

\section{Cold-Head Unit}

The gas is cooled down in the cold-head unit consisting of two stages on which two copper heat exchangers are mounted. Because the heat capacity of copper is large, a quasi-stationary profile of the gas in both heat exchangers is assumed. Also from experimental data the $\mathrm{Mc}_{\mathrm{v}}$ term appears to be negligible compared to the $\dot{m} c_{\mathrm{p}}$ term in eq. (1). The resulting stationary equation for the gas in the heat exchanger $\mathrm{T}_{\mathrm{ci}}(\mathrm{x})(\mathrm{i}=1,2$ for the first and second stage) can be solved analytically. Here $\mathrm{x}$ (in $\mathrm{m}$ ) is the position coordinate. Because of the high thermal conductivity of the cold-head stages, a uniform material temperature $T_{m c i}$ is assumed. Equation (2) can now be written (in $W$ ) as

$$
M_{m c i} C \frac{d T_{m c i}}{d t}=\int h P_{i}\left(T_{c i}-T_{m c i}\right) d x-k_{i} \dot{q}_{C i}\left(T_{m c 1}, T_{m c 2}\right)
$$

in which the integration is carried out over the length $L_{i}$ (in $m$ ) of the heat exchanger channel. Here $\mathrm{M}_{\mathrm{mci}}=$ the total mass of the cold head plus heat exchanger $(\mathrm{kg})$, $\dot{\mathrm{q}}_{\mathrm{ei}}=$ specified cooling power of the stage (W) in principle depending on both cold head temperatures $T_{m c 1}$ and $T_{m c 2}$, and $k_{i}=$ efficiency of the cold head. Substitution of the analytical expression of $T_{\mathrm{ci}}$ in eq. (3) results in

$$
M_{m c i} C \frac{d T_{m c i}}{d t}=\dot{m} C_{p}\left(T_{c i}(x=0)-T_{m c i}\right)\left(1-e^{-\frac{h_{i} P_{i} L_{i}}{\dot{m} C_{p}}}\right)-k_{\dot{i}} \dot{q}_{C i}\left(T_{m c i}\right)
$$

in which $\mathrm{T}_{\mathrm{cij}}(\mathrm{x}=0)=$ incoming gas temperature. Based on the cooler specifications, $\dot{\mathrm{q}}_{\mathrm{ci}}$ is assumed to be a function of $\mathrm{T}_{\mathrm{mci}}$ only [3]; $\dot{\mathrm{q}}_{\mathrm{cl}}$ is fitted to a fourth order polynomial and $\dot{\mathrm{q}}_{\mathrm{c} 2}$ to a fifth. The heat transfer coefficient $h$ can be written as $h=\lambda \cdot \mathrm{Nu} / \mathrm{D}_{\mathrm{h}}$, in which $\mathrm{Nu}=0.00222 \cdot \operatorname{Pr}^{0.4} \cdot \operatorname{Re}^{1.09}[4], \lambda=$ heat conductivity of helium gas $\left(\mathrm{Wm}^{-2} \mathrm{~K}^{-1}\right)$, and $\mathrm{D}_{\mathrm{h}}=$ hydraulic diameter of the channel $(\mathrm{m})$. The Prandtl-number of helium gas $\mathrm{Pr}$ is assumed constant at 0.704 , whereas the Reynolds-number $\operatorname{Re}$ can be written as $\operatorname{Re}=4 \dot{\mathrm{m}} /(\mu \mathrm{P})$ 
in which $\mu=$ dynamic viscosity of helium (in $\mathrm{kgs}^{-1} \mathrm{~m}^{-1}$ ). $\lambda$ and $\mu$ are fitted by $\lambda=1.875 \cdot 10^{-3} \mathrm{~T}^{0.835}$ and $\mu=$ $5.335 \cdot 10^{-7} \mathrm{~T}^{0.641}, c_{\mathrm{p}}=5.2 \cdot 10^{3}$ and $\mathrm{c}$ is fitted by a third order polynomial. $\mathrm{D}_{\mathrm{h}}=1.5 \cdot 10^{-3}, \mathrm{P}_{\mathrm{i}}=6 \cdot 10^{-3}, \mathrm{~L}_{1}=$ 0.7 and $\mathrm{L}_{2}=1$, whereas $\mathrm{M}_{\mathrm{mcl}}$ and $\mathrm{M}_{\mathrm{mc2}}$ are estimated at 0.6 and 0.15 , respectively. Assuming that the cooldown curve of $T_{C F c o}$ equals $T_{c 1}(x=0)$ and the calculated $T_{m c 1}$ equals $T_{c 2}(x=0)$, the model eq. (4) was integrated numerically by the Runge-Kutta method, resulting in best fit values $k_{1}=0.7$ and $k_{2}=0.6$. A comparison of model and measurements at $\dot{m}=1.4 \cdot 10^{-5} \mathrm{kgs}^{-1}$ is presented in Fig. 2. The fact that the $\mathrm{k}_{\mathrm{i}}$ values are below 1 may be caused by aging of the cooler ( 11 years), especially concerning the sealing around the displacers in the cold heads.

\section{Rest of the System}

In the counterflow heat exchanger the temperature of the outcoming (supply) gas at the cold side $\left(\mathrm{T}_{\mathrm{CF} \text { co }}\right)$ can be expressed in that of the incoming cold (return) gas ( $\mathrm{T}_{\mathrm{CF} \mathrm{i}}$ ) by means of an efficiency $\eta=$ $\left(\mathrm{T}_{\mathrm{e}}-\mathrm{T}_{\mathrm{CFco}}\right) /\left(\mathrm{T}_{\mathrm{e}}-\mathrm{T}_{\mathrm{CFci}}\right)$. Here $\mathrm{T}_{\mathrm{e}}$ is the temperature at the warm side assumed to be that of the environment. For $T_{c}=294 \mathrm{~K}$ a value $\eta=0.922$ results from the measurement data. The rest of the system (between cold head and $\mathrm{CFci}$ ) is described by a model based on ideal heat exchange between gas and material resulting in $T=T_{m}$. Three elements are treated separately: the supply line, the SQUID unit (considered as a single heat exchanger) and the return line. The external heat load is assumed to be determined by radiative flow from the environment at $294 \mathrm{~K}$ (also for the supply line). The equations (1) and (2) yield

$$
\left(M C_{V}+M_{m} C\right) \frac{\partial T}{\partial t}+\dot{m} C_{p} \frac{\partial T}{\partial X}=k\left(T_{e}^{4}-T^{4}\right)
$$

in which $\mathrm{k}$ is a heat transfer coefficient. Equation (5) is used for all three elements. The heat transfer coefficients $\mathrm{k}$ have been estimated from the stationary temperature distributions. The resulting values (in $\mathrm{Wm}^{-1} \mathrm{~K}^{-4}$ ) are: supply line $5.15 \cdot 10^{-11}$, SQUID unit $9.42 \cdot 10^{-11}$, and return line $1.09 \cdot 10^{-10}$. The mass-timesheat-capacity term (in $\mathrm{Jm}^{-1} \mathrm{~K}^{-1}$ ) reduces for decreasing temperature for the supply line from about 4 to 0.07 , for the SQUID unit from about 20 to 2.5 , and for the return line from roughly 50 to 7 . The gas contribution $\mathrm{Mc}_{\mathrm{v}}$ has a maximum value of typically $2 \cdot 10^{-3} \mathrm{Jm}^{-1} \mathrm{~K}^{-1}$ and is therefore negligible. In the model we assumed the factors $\mathrm{M}_{\mathrm{m}} \mathrm{c}$ to be constant and fitted their values to the experimental data. The best results were obtained with the following values (in $\mathrm{Jm}^{-1} \mathrm{~K}^{-1}$ ): supply line 11, SQUID unit 15, and return line 40. A comparison of model and measurements for a flow of $1.4 \cdot 10^{-5} \mathrm{kgs}^{-1}$ is presented in Fig. 3. The deviations are obviously due to neglecting the temperature dependancy in $M_{m} c$. At high temperatures the real capacity is higher than in the model and, therefore, cooldown is slower, whereas at low temperatures the real capacity is lower and as a result the cooldown in the real situation is faster than in the model. The next step in our model development will be the incorporation of this temperature dependancy. Also the supply line has to be considered in more detail because the model value of $\mathrm{M}_{\mathrm{m}} \mathrm{c}$ is quite high and also the assumption that the external load is radiation from $294 \mathrm{~K}$ is not very realistic.

\section{DISCUSSION}

A model is developed to describe the thermodynamic behaviour of our closed-cycle gas flow system. It describes two subsystems. The model for the cold-head unit gives sufficiently good results. The model for the rest of the system has to be improved. In this respect we will first focus on the temperature dependancy of the heat capacities involved. As a final step the cold-head model will be combined to that of the rest, so as to obtain a description of the complete system.

\section{REFERENCES}

I van den Bosch, P.J., Holland, H.J., ter Brake, H.J.M. and Rogalla, H. Operation of a closed-cycle gas flow system for cooling a $\mathrm{HT}_{\mathrm{c}}$ dc-SQUID magnetometer, to be published in Cryogenics

2 van den Bosch, P.J., de Boer, H.A., Holland, H.J., ter Brake, H.J.M. and Rogalla, H. The application of cryocoolers for cooling a high- $T_{6}$ SQUID magnetometer, to be presented at 8 th Intern. Cryocooler Conf. at Vail, CA in June 1994

3 Operation Instructions of Kryo-Refrigeratoren, Leybold-Heraeus GMBH, Köln, Germany (1981) and 
private communication

4 Wu Peiyi, Little, W.A., Measurement of the heat transfer characteristics of gas flow in fine channel heat exchangers used for microminiature refrigeration, Butterworths (1984)

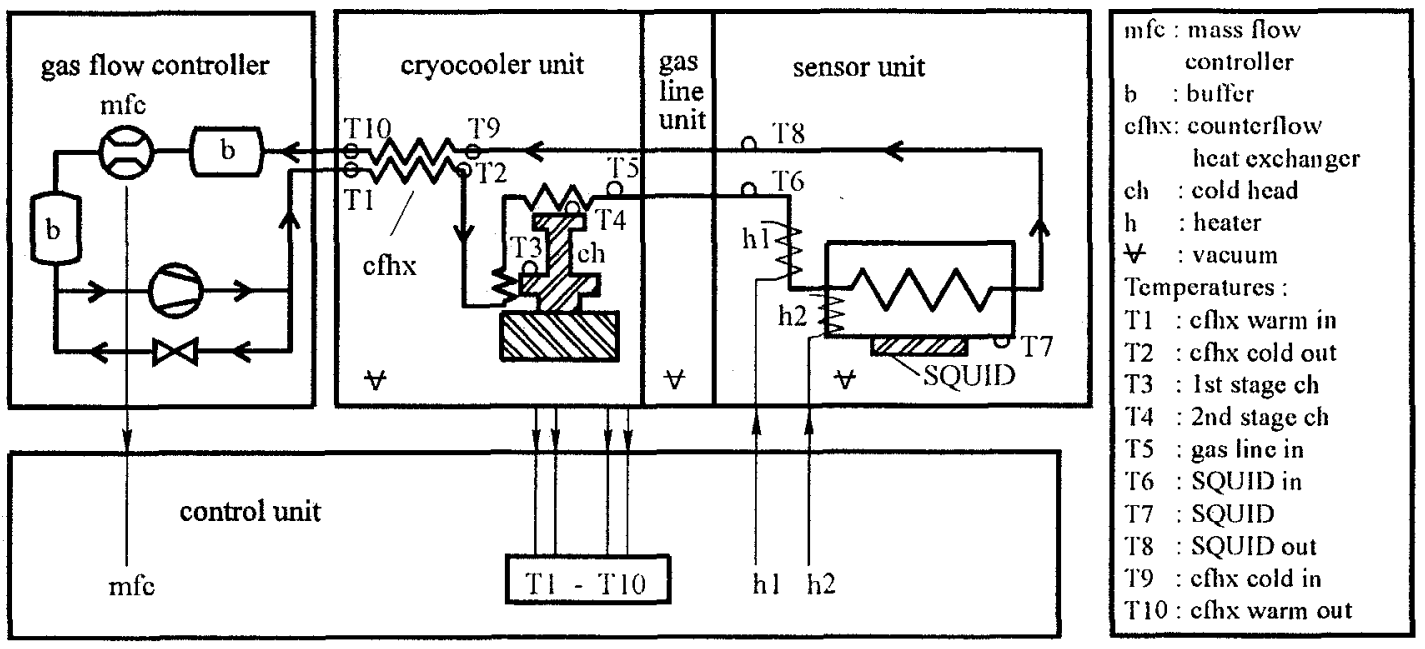

Figure 1 Diagram of the closed-cycle gas flow system.

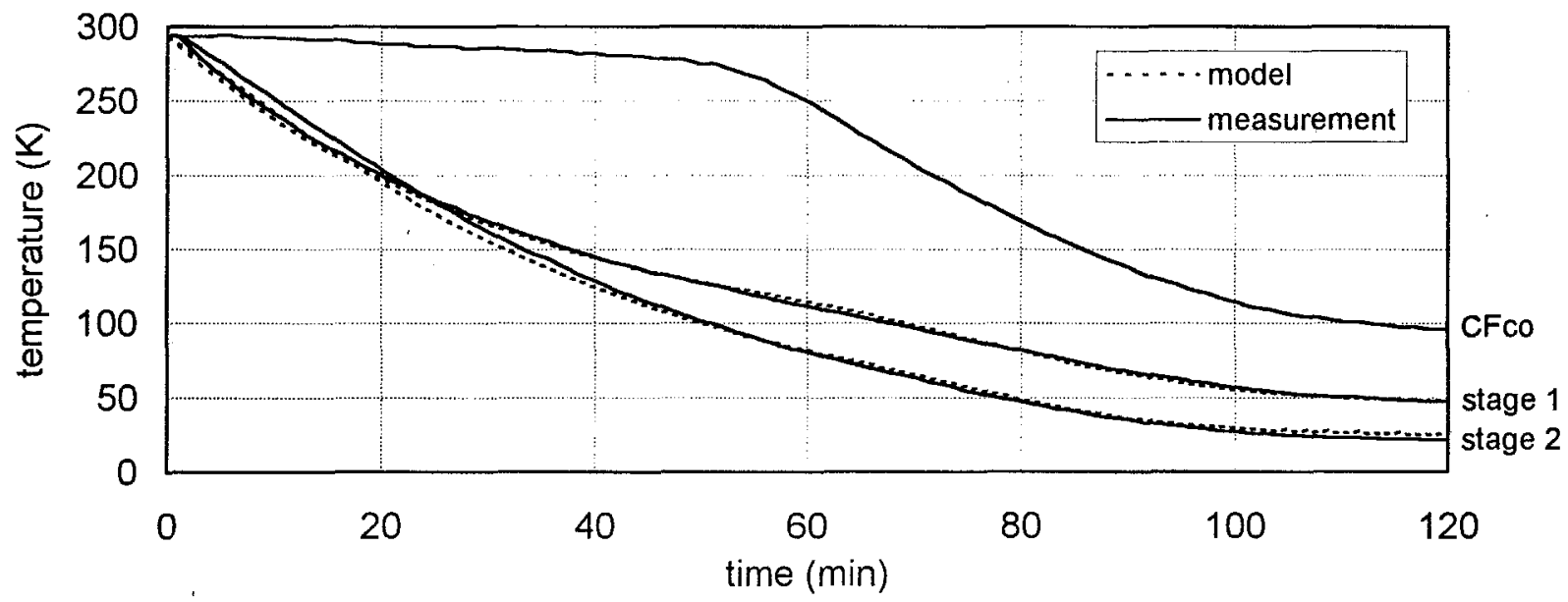

Figure 2 Comparison of the cold-head unit model and measurements at $\dot{\mathrm{m}}=1.4 \cdot 10^{-5} \mathrm{kgs}^{-1}$.

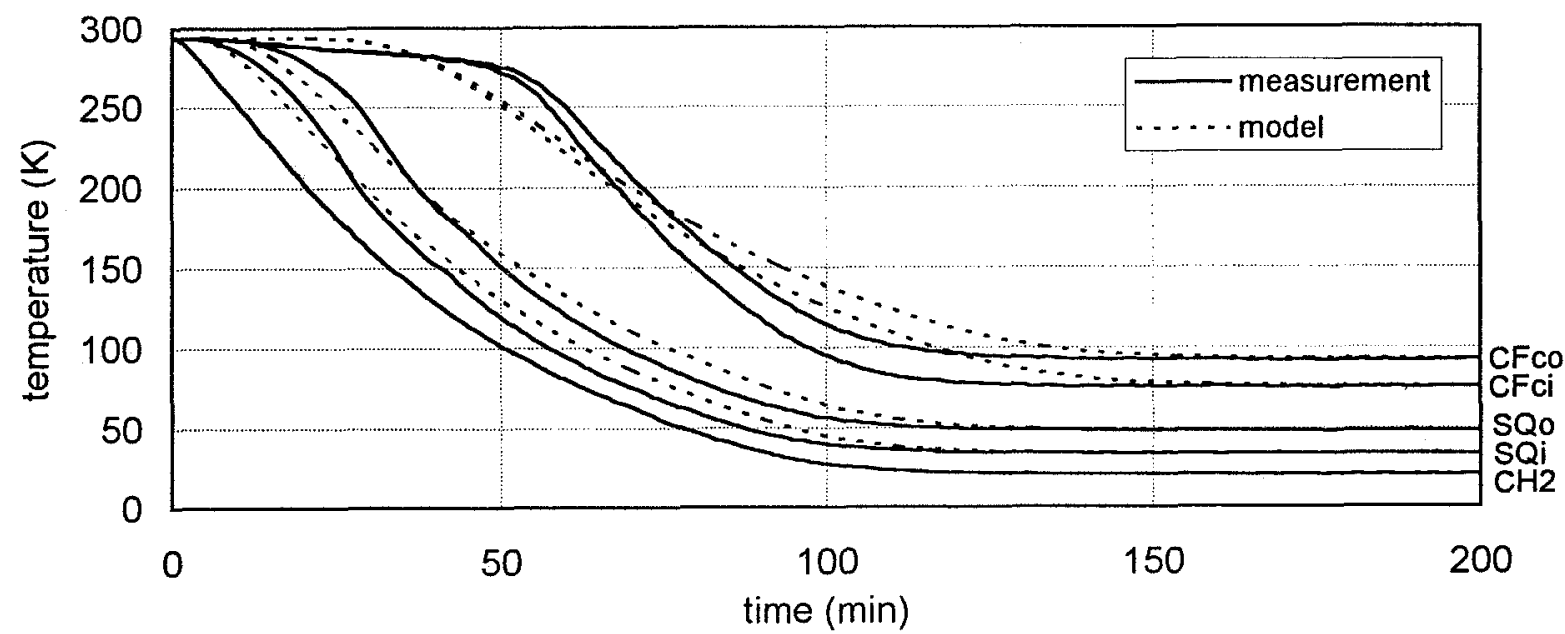

Figure 3 Comparison of the rest model and measurements at $\dot{\mathrm{m}}=1.4 \cdot 10^{-5} \mathrm{kgs}^{-1}$. SQi and SQo denote the inlet and outlet temperature of the SQUID unit, $\mathrm{CH} 2$ the second stage temperature. 
Cooling systems for HTS electronics and cryoelectronic components

Armin Binneberg, Hanno Buschmann, Johannes Neubert, Gabriele Spörl

Institut für Luft- und Kältetechnik, Bertolt-Brecht-Allee 20, 01309 Dresden, Germany

\begin{abstract}
HTS components require that low-cost, reliable cooling systems be used. There are no general solutions to such systems. Any cooling concept has to be tailored to the specific requirements of a system. The ILK Dresden has special cryostats develops for SQUID gradiometers, for superconductive high frequency systems and for diodes lasers (environment monitoring). At examples informed about design-data and design-philosophy.
\end{abstract}

\title{
INTRODUCTION
}

The development and construction of cryostats is a complicated task. Fundamental questions must clarified his to solution of the problems. The following has to be taken into consideration when designing cooling concepts:

1. Cooling temperature

2. Constancy and controllability of the cooling temperature

3. Cooling load and refrigerating capacity

4. Continuous or discontinuous mode

5. Dregree of automation

6. Life time respectively availability of the cryocooler

7. Malfunctions caused by microphonic, thermal or electromagnetic effects

8. Stationary or mobile application

9. Investment and operating costs

Furthermore is important: Selection of the material and processing of the material. The manufacture of cryostats requires special technological skills and know-how.

\section{Examples: bath cryostats, cryostats with refrigerators and new cooling systems}

If, for example, the magneto cardiogram has to be measured in an unshielded atmosphere using a SQUID gradiometer system the cooling system has to meet the following requirements:

- cooling temperature

$80 \mathrm{~K}$

- max. cooling temperature variation

$\pm 0,01 \mathrm{~K}$

- refrigerating capacity

approx. $1 \mathrm{~W}$

- continuous measuring

- no vibrations

- non-metallic construction

- max. distance between sensor and test object $\quad 6 \mathrm{~mm}$

In order to meet the above requirements the cryostat shown in Figure 1 was developed, and a small number manufactured. 
The whole cryostat is made of glass-fibre reinforced epoxy resin materials and has glued lowtemperature resistant, vacuum-tight seams and an integrated cryopump. It has the following features:

- $\mathrm{LN}_{2}$ capacity

5 litres

- $\mathrm{LN}_{2}$ evaporating rate

$20 \mathrm{ml} / \mathrm{h}$

- full serviceability before evacuation:

1 month

A cryostat meeting the following requirements was needed no measure the thermal and electrical properties of superconductive materials in dependance on temperature and the magnetic field:

- cooling temperature

$4,2 \ldots 300 \mathrm{~K}$

- constancy of cooling temperature

$$
\pm 0,1 \mathrm{~K}
$$

- refrigerating capacity

$0 \ldots .5 \mathrm{~W}$

- continuous measuring

- low vibration level

- non-metallic materials in the measuring area

- magnetic field

$0 \ldots 3$ Tesla

A schematic representation of the cryostat manufactured to meet the above requirements is shown in Figure 2.

The outer Dewar vessel contains liquid helium and a superconductive coil to generate the magnetic field. The test object is arranged inside the Dewar system. The helium flow and electric fine controlling systems permit the test object to be cooled down to any temperature between $4,2 \mathrm{~K}$ and $300 \mathrm{~K}$ the controlling accuracy being $0,1 \mathrm{~K}$. The superconductive magnet can be used to generate magnetic fields within a range of 0 Tesla to 3 Tesla so as to enable the material properties to be analyzed depending on the applied outer magnetic field.

Before it will be possible to apply devices with high-temperature superconductive components outside research laboratories on a broad scale it is necessary to go over from liquid cooling to mechanical, i.e. low-maintenance, cooling first.

As you need special cryostats for liquid cooling so you need specially designed cooling devices for gas refrigerating machines. These devices have to take into account the specific requirements of HTS components.

A demonstrator became develops to the cooling of HTS aerials. It includes a compressor box and a Dewar system sheathing the cold head of the Stirling machine. The compressor box comprises the split Stirling machine compressor, an electronic control device and a ventilator for compressor cooling. The Dewar system has to he designed and constructed with much care to ensure that the HTS aerials are actually cooled down to about $85 \mathrm{~K}$ using the available refrigerating capacity of $1,2 \mathrm{~W}$. The sample mount is provided with a thermometer and a heater. The Dewar system has weak-current and HF bushings so that the corresponding lines can be connected. The demonstrator can be completed by a temperature controller to permit any desired sample temperature between $60 \mathrm{~K}$ and $300 \mathrm{~K}$ to be set. It is possible to connect the aerial mount directly to the cold head of the Stirling machine because HTS aerials have to be cooled down to no specific but any temperature below the transition point, $T_{c}$, and because they are relatively insensitive to vibration. The self-contained, mobile sensor cooler can be applied wherever it is needed. 
A low-trouble and discontinuous operating SQUID cooling system was developed. A schematic representation is given in Figure 3.

Here again a split Stirling machine is used to generate cold. Its cold head is connected to the capillary store.

The Stirling machine causes nitrogen to be liquefied in the solid matrix of the capillary store. The object to be cooled is thermally coupled to the capillary store. SQUID cooling is almost troublefree because liquid nitrogen evaporates only when the Stirling machine is not running. As soon as the whole amount of nitrogen has evaporated it is liquefied again by the Stirling machine via a regenerator.

The problem of this cooling concept lies in keeping the pressure and thus the temperature constant in a closed nitrogen circuit. We are presently working on this problem. A possible version is shown in the figure. This cooling system is to ensure a 10-hour low-trouble measurement using a refrigerating capacity of about $0,7 \mathrm{~W}$ which is required for cooling the SQUID and its supply lines at $80 \mathrm{~K}$. To liquefy nitrogen the Stirling machine has to be in operation for approximately 1 hour.

Should the refrigerating capacity or the cooling temperature of about $80 \mathrm{~K}$ not be sufficient it is possible to use the regenerator mass of the second stage of a Gifford-McMahon machine as a cold-generating and storing element.

The Jena Friedrich Schiller University is working on designing a cooling system of that type.

If materials are to be tested by HTS SOUIDs, e.g. in an aircraft, a low-trouble cooling system is required working independently of its position. This is necessary because the construction contours of the aircraft have to be scanned by the SQUID measuring head. One possible construction of a cooling system of this type is shown in Figure 4.

The cooling system includes a portable nitrogen replenishing container of an $\mathrm{LN}_{2}$-replenishing line and a SOUID cooling head.

The SQUID cooling head is designed so as to ensure low-trouble measurement with a filling quantity of 0,51 for about 3 hours. The cooling head can be turned to any position without liquid nitrogen escaping from the gas outlet and without preventing the SQUID from being cooled properly. To this end the $\mathrm{LN}_{2}$ replenishing line and the $\mathrm{N}_{2}$ waste gas line are arranged coaxially and are brought to the centre of the $\mathrm{LN}_{2}$ filling space. The filling space is filled with bronze wiremesh screen cloth to ensure good thermal contact. 


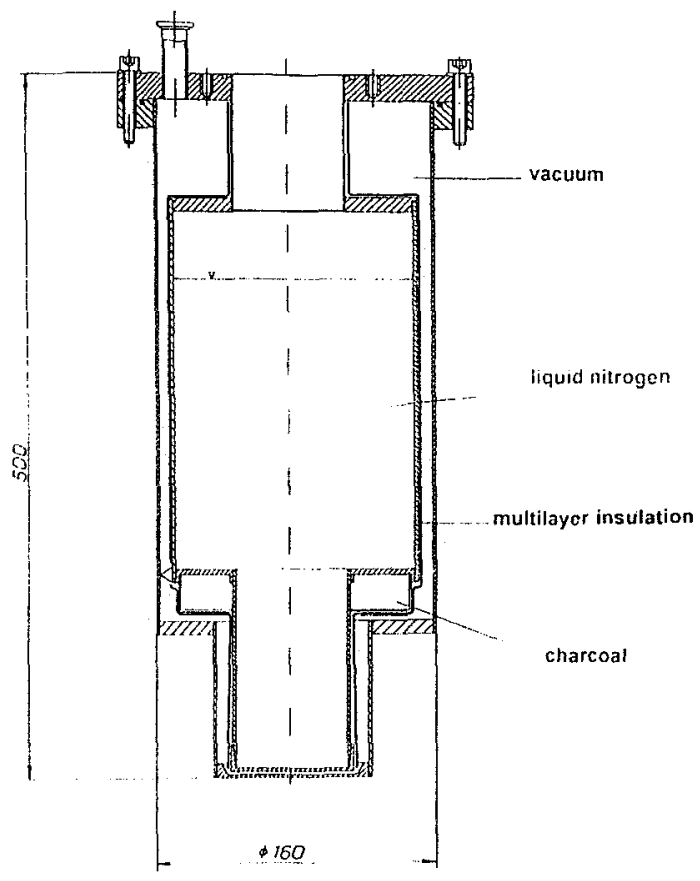

Fig. 1: Cryostat for SQUID COOLING

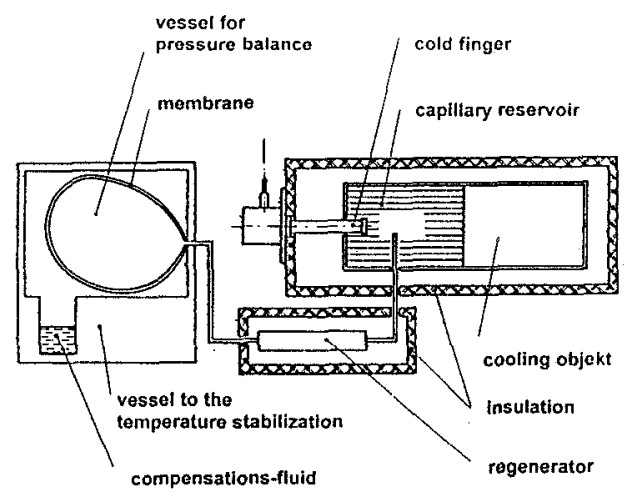

Fig. 3: Diagram to the alternating reservoir cooling

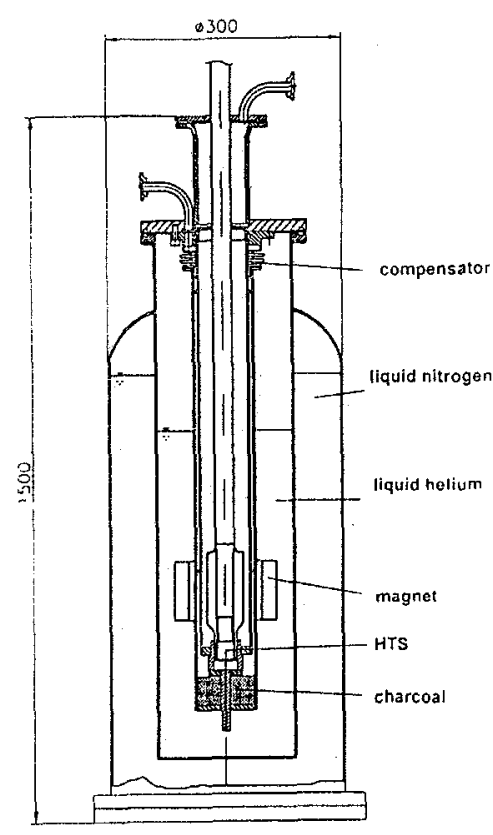

Fig. 2: Cryostat to the analysis of thermal and electric material characteristics

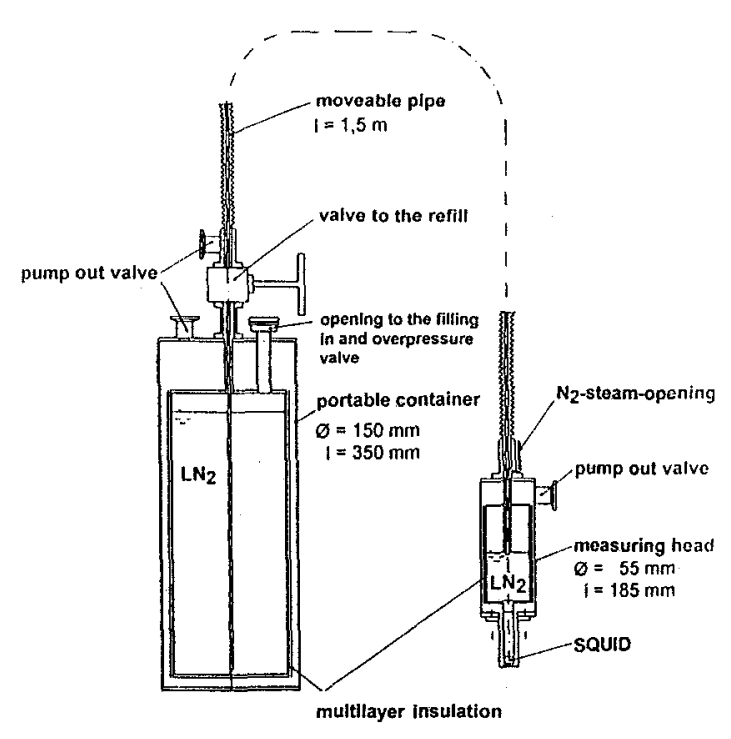

Fig. 4: Portable SQUID-COOLING-SYSTEM 